Planet (234) Barbara.

\begin{tabular}{|c|c|c|c|c|c|c|c|c|c|}
\hline 5883 & M.Z.Prag & $\Delta \alpha$ & $\Delta \delta$ & Vgl. & $\alpha$ app. & $\log p \cdot A$ & $\delta$ app. & $\log p \cdot A$ & * \\
\hline Oct. & $9^{\mathrm{h}} 6^{\mathrm{m}} 10^{\mathrm{s}}$ & $-0^{\mathrm{m}} 46.60$ & $+8^{\prime} 38^{\prime \prime} 8$ & Io & $21^{\mathrm{h}} \times 8^{\mathrm{m}} 22^{\mathrm{s}} .0 \mathrm{I}$ & 7.753 & $-24^{\circ} 10^{\prime} 30^{\prime \prime} 9$ & $9.98 \mathrm{I}$ & 3 \\
\hline 2 & 926 I 6 & +o 0.29 & +310.2 & 5 & $\begin{array}{lll}2 \text { I } & \text { I } 9 & 9.0 \text { I }\end{array}$ & 8.012 & $\begin{array}{lll}-24 & 15 & 59.7\end{array}$ & 9.979 & 3 \\
\hline
\end{tabular}

Mittlerer Ort des Vergleichsternes.

\begin{tabular}{|c|c|c|c|}
\hline$*$ & $a+883.0$ & $\delta$ I 883.0 & Autorität \\
\hline 3 & $21^{\mathrm{h}} 19^{\mathrm{m}} \quad 4^{\mathrm{s}} \div 58$ & $-24^{\circ} \mathrm{I} 9^{\prime} 3 \mathrm{I}^{\prime \prime} \mathrm{O}$ & B.A.C. $743^{6}$, Yarnall $935^{8}$ \\
\hline
\end{tabular}

Erste Beob. durch: Weinek; zweite durch: Weinek und Gruss.

Der Planet erschien 10.5 Grösse; am 2. October war er wegen ungünstiger Luft und der grussen Nähe eines Sternchens gleicher Grösse schwer zu beobachten.

Prag $188_{3}$ Oct. Io.

L. Weinek.

Z u s a $\mathrm{z}$. In Nr. 2546 pag. 27 finden sich neuere Bestimmungen des Sternes 1 ; der zweite, AOe. 16243 , findet sich ebenfalls in meinen Zonen:

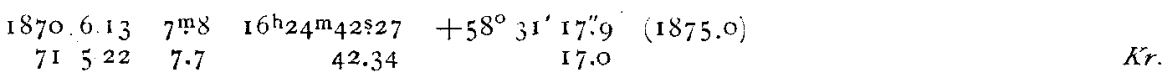

\title{
Elliptic Elements of Comet 1882 I.*)
}

The accompanying set of elements was derived from the following six normal places:

\begin{tabular}{|c|c|c|c|c|c|c|c|}
\hline G. M. Т. & $\begin{array}{c}\text { Mean RA. } \\
\text { I } 882.0\end{array}$ & $\begin{array}{c}\text { Probable } \\
\text { Error }\end{array}$ & Obs. & $\begin{array}{ll} & \text { Mean } \\
\delta \quad 1882.0\end{array}$ & $\begin{array}{c}\text { Probable } \\
\text { Error }\end{array}$ & Obs. & Time of normal places \\
\hline Mar. 26.5 & $27 x^{\circ} 36^{\prime} 46^{\prime \prime} 9$ & \pm 0.71 & $6_{3}$ & $37^{\circ}$ I $5^{\prime}$ I $5^{\prime \prime} \cdot 6$ & $\pm 0.4 I$ & $6 !$ & Mar. I $9-$ Apr. \\
\hline April 1 2.5 & $281 \quad 22 \quad 16.7$ & \pm 0.20 & 70 & 4949 I 5.1 & \pm 0.66 & 70 & Apr. $\quad 4-$ Apr. 2 I \\
\hline May 25.5 & $\begin{array}{lll}55 & 55 & 34.2\end{array}$ & \pm 0.45 & 40 & $\begin{array}{lll}59 & 3^{6} \quad 4.1\end{array}$ & \pm 0.28 & 40 & May $2 \mathrm{I}-$ May 28 \\
\hline June $\quad 2.5$ & $\begin{array}{lll}66 & 45 & 42.3\end{array}$ & \pm 0.56 & 18 & $443^{6} \quad 27.1$ & \pm 0.53 & I 7 & June 1 -June 5 \\
\hline July & I $56 \quad 59 \quad 16.3$ & \pm 0.59 & 27 & $\begin{array}{lll}9 & 45 & 17.5\end{array}$ & $\pm r \cdot 3$ & 27 & 8 - July $\quad 17$ \\
\hline Aug. 7.5 & I 8 I $46 \quad 55.5$ & \pm 1.17 & I 2 & $\begin{array}{lll}4 & 14 & 3.7\end{array}$ & $\pm 1 \cdot 3$ & I 2 & Aug. I- Aug. I 6 \\
\hline
\end{tabular}

From the $2^{\mathrm{d}} 3^{\mathrm{d}}$ and $5^{\text {th }}$ of these normal places a preliminary set of elements was computed according to the methods given in Gauss' >Theoria Motus \& as follows:

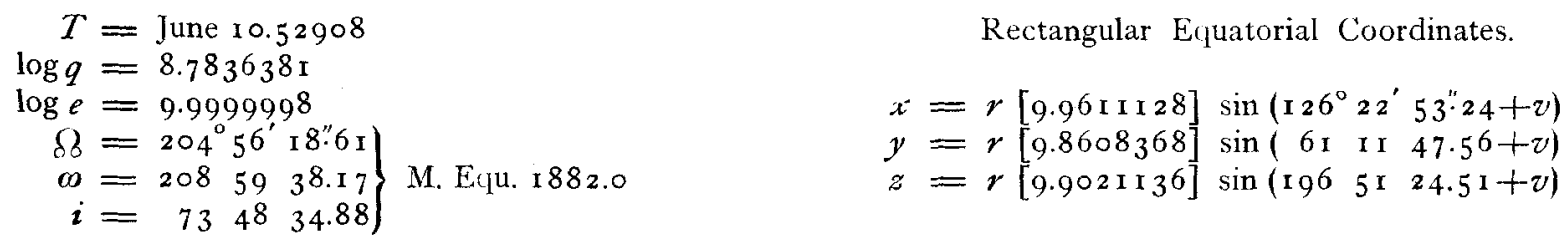

With these elements considering the excentricity equal to unity comparison was made with all six normal places, in order to make the final corrections to the preliminary orbit by means of a least square solution.

*) Prof T. H. Safford schreibt mir in Bezug auf die Aufforderung in Nr. 2526, dass der Verfasser obigen Artikels schon seit längerer Zeit nach Rüicksprache mit Prof. L. Boss die Berechnung des Cometen übernommen habe und dass seine Rechnungen zur Zeit des Erscheinens der erwähnten Aufforderung so weit vorgeschritten gewesen seien, dass er dieselben auch $z \mathbf{u}$ Ende $z \mathbf{u}$ führen wünschen würde, $K r$. 
A computation in duplicate by two independent methods gave the following twelve equations of condition given in logarithmic form.

\begin{tabular}{|c|c|c|c|c|c|c|c|}
\hline$(\boldsymbol{x})$ & $(y)$ & $(z)$ & (u) & $(v)$ & $(w)$ & $(n)$ & \\
\hline $9.16489 n$ & 9.27446 & 9.95933 & $9.08991_{n}$ & $9.465 \mathrm{r} 8$ & $9.44609 \mathrm{n}$ & 0.27560 & $=0$ \\
\hline $8.41902 \mathrm{n}$ & 8.98582 & 9.70896 & 9.97296 & $9.83479_{11}$ & 9.81569 & 0.72835 & $=$ \\
\hline $9 \cdot 3^{6} 39 \mathrm{In}_{\mathrm{n}}$ & $9 \cdot 34675$ & $9.9790 \mathrm{I}$ & $9.5^{2692}$ & 9.31175 & $9 \cdot 322$ I $2 \mathrm{n}$ & $8.5^{8} 790$ & $=$ \\
\hline $8.75669 n$ & 8.80395 & 9.44677 & 8.46007 & $9.87899 \mathrm{n}$ & $9.8893^{6}$ & 9.73240 & $=$ \\
\hline 8.47717 & $9.1994^{2}$ & $9.68374 n$ & $9.84899 \mathrm{n}$ & $9.39972_{n}$ & $9.6 \times 464$ & $0.16810_{n}$ & $=$ \\
\hline 9.84246 & $9.05^{28} 3_{11}$ & $9.23382_{n}$ & 9.84595 & $7.49914 \mathrm{n}$ & 7.71406 & $0.127 \mathrm{IO}_{\mathrm{n}}$ & $=$ \\
\hline 9.075 I 2 & $9 . \mathrm{I}_{437 \mathrm{I}_{\mathrm{n}}}$ & $9.4888 \mathbf{I}_{\mathrm{n}}$ & $9.4823^{8} n$ & $8.97764 n$ & $9.375^{6} 3$ & $0.48 \times 50_{n}$ & $=$ \\
\hline 9.91297 & $8.85{ }^{6} 65 n$ & $8.76015 n$ & $9.49 \times 19$ & $8.15680_{n}$ & 8.55479 & $0.47860_{n}$ & $=$ \\
\hline $9.497^{6} 4 n$ & $9.12382_{n}$ & $9 \cdot 5^{8} 5^{22}$ & 9.60400 & $9.4405^{8}$ & 8.86482 & $9.03510_{n}$ & $=$ \\
\hline $8.3^{88} 59$ & $9.19260_{n}$ & 9.78999 & $0.11026_{n}$ & $0.44974 n$ & $8.87398_{n}$ & $9.23040 n$ & $=$ \\
\hline $8.99356_{\mathrm{n}}$ & $8.9962 \mathrm{I}_{\mathrm{n}}$ & $9.629^{6} 3$ & $8.8 \simeq 397$ & 9.61626 & $8.7 \times 295$ & $0.65390_{11}$ & $=$ \\
\hline $7.44648_{n}$ & $9.07909 \mathrm{n}$ & 9.77796 & $0.16787 \mathrm{n}$ & $9.52880_{n}$ & $8.62549 n$ & 0.78890 & $=$ \\
\hline
\end{tabular}

where:

$$
\begin{aligned}
x & =10000 \mathrm{~d} T \\
\mathrm{M} \sin y & =\mathrm{d} \log q \text { (Briggian) or } y=\frac{\mathrm{d} q}{q \sin \mathrm{I}^{\prime \prime}} \\
z & =\mathrm{d} \chi^{\prime}=\mathrm{d} o^{\prime}+\cos i^{\prime} \mathrm{d} \Omega^{\prime} \\
\sin u & =\mathrm{d} e \text { or } \mathrm{d} e=\frac{u}{\sin \mathrm{I}^{\prime \prime}} \\
a & =\mathrm{d} \Omega^{\prime} \\
\omega & =\mathrm{d} i^{\prime}
\end{aligned}
$$

It was not thought worth while to apply weights systematically according to the number of observations, but as the last normal place seemed to have considerably less precision than the others, the last two equations were given a weight of $0.50 \mathrm{r} 2, \log 9.70000$, as a convenient approximation to a weight of one half. The above equations of conditions give the following normal equations.

$$
\begin{array}{r}
1.3521 \%-0.2004 y-0.7279 z+0.5948 u-0.1752 v+0.0742 z-4.0124 n=0 \\
-0.2004+0.2147+0.3884+0.2805+0.0471-0.0861+1.8309=0 \\
-0.7279+0.3884+3.2376-0.7158-0.0294-0.1974+7.4300=0 \\
+0.5948+0.2805-0.7158+5.1019+0.1672+0.5524+0.3768=0 \\
-0.1752+0.0471-0.0294+0.1672+1.5376-1.2257-4.7598=0 \\
+0.0742-0.0861-0.1974+0.5524-1.2257+1.3913+1.7076=0
\end{array}
$$

Giving the following values for the unknown quantities:

$$
\begin{aligned}
& x=4.477 \mathrm{I} \pm \mathrm{r} \cdot 468 \\
& y=2.4213 \pm 4.2 \times 4 \\
& z=-\mathrm{r} .4295 \pm 0.875 \\
& u=-2.2662 \pm 0.877 \\
& \because=+10.95^{2} \pm 2.495 \\
& w=+9.035 \quad \pm 2.739
\end{aligned}
$$

Substituting these values in the equations of condition gives for the sum of the squares of the residuals $[v v]=$ 22.02. In the solution of the normal equations $[n n .6]=$ 22.09 .

From these unknown quantities the following corrections to the preliminary elements are found:

$$
\begin{array}{rlrl}
\mathrm{d} T & =+0.000448 & \pm 0.000147 \\
\mathrm{~d} \log \eta & =+0.000005 \mathrm{I} & \pm 0.0000089 \\
\mathrm{~d} \omega^{\prime} & =-8.024 & & \pm 2.644 \\
\mathrm{~d} e & =-0.00001098 & \pm 0.00000425 \\
\mathrm{~d} \delta^{\prime} & =+10^{\prime \prime}=5^{\prime \prime} & & \pm 2.495 \\
\mathrm{~d} i^{\prime} & =+9.035 & & \pm 2.739
\end{array}
$$

These corrections give as the most probable values of the elliptic elements:

$$
\begin{aligned}
& T=\text { June } 10.52953 \text {. G. M. 'T. } \pm 0.00015 \\
& \log q=8.783643^{2} \quad \pm 0.0000089 \\
& e=0.99998902 \pm 0.00000425
\end{aligned}
$$




$$
\begin{aligned}
& \omega=208^{\circ} 59^{\prime} 33^{\prime \prime} \cdot 72 \text { Mean Equinox } \\
& \delta=2045629.49 \text { and Ecliptic } \\
& i=\begin{array}{lll}
73 & 48.82
\end{array} \quad \text { I } 882.0 \\
& \left.\omega^{\prime}=1965 \text { I } 16.49 \pm 2.644\right) \text { Referred } \\
& \left.\delta^{\prime}=21029 \text { r } 2.25 \pm 2.495\right\} \text { to } \\
& i=5^{2} 5741.04 \pm 2.739 \text { Equator }
\end{aligned}
$$

Rectangular Equatorial Coordinates.

$$
\begin{aligned}
& x=r[9.9611023] \sin \left(126^{\circ} 22^{\prime} 49^{\prime \prime} 33+v\right) \\
& y=r[9.8608362] \sin (6 \text { I I I } 58.04+v) \\
& z=r[9.9021280] \sin \left(\begin{array}{llll}
196 & 51 & 16.49+v)
\end{array}\right.
\end{aligned}
$$

\begin{tabular}{|c|c|c|c|c|}
\hline & $\begin{array}{c}d a \cos \phi \\
(c--o)\end{array}$ & 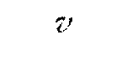 & $\begin{array}{c}\mathrm{d} \delta \\
(\mathrm{c}-\mathrm{o})\end{array}$ & $z^{\prime}$ \\
\hline Mar. 26.5 & $+1 " 36$ & $+1 " 34$ & $+I " O I$ & $+\mathrm{I} .00$ \\
\hline April I 2.5 & -0.86 & $-0.7 \mathrm{I}$ & $-I \cdot 3 I$ & -1.31 \\
\hline May 25.5 & +1.53 & $+1.5^{2}$ & +0.19 & +0.17 \\
\hline June 2.5 & $-0.6 \mathrm{I}$ & -0.60 & +0.04 & +0.03 \\
\hline July & +0.37 & +0.38 & -2.33 & -2.16 \\
\hline Aug. 7.5 & $-0.9 \mathrm{I}$ & -0.95 & $+4.3^{\circ}$ & +4.24 \\
\hline
\end{tabular}

These elements correspond to a period of about 400000 years.

Recomputing the position of the comet for the dates of the 6 normal places and comparing, gives the following

Field Memorial Observatory, Williamstown, Mass. results. The column headed $z z:$ being the residuals obtained by substituting in the equations of condition the values found above for the unknown quantities.

\begin{tabular}{|c|c|c|c|c|c|c|c|c|c|}
\hline $188_{3}$ & M. Z. Hels. & $\Delta \alpha$ & $\Delta d$ & Vergl. & $\alpha$ app. & $\log p .1$ & $\delta$ app. & $\log p .4$ & $*$ \\
\hline Oct. 6 & $8^{\mathrm{h}} 5^{8^{\mathrm{m}}} 34^{\mathrm{s}}$ & $+0^{\mathrm{m}} \times 5^{\mathrm{s}} \cdot 68$ & $+\circ 48.1$ & 5 & $16^{\mathrm{h}} 30^{\mathrm{m}} 21 \mathrm{~s} \cdot 50$ & $9 \cdot 740$ & $+57^{\circ} 45^{\prime}$ II I" I & 0.555 & I \\
\hline 10 & $92 \mathrm{I} \quad 2 \mathrm{I}$ & + I $\quad 9.70$ & + $10 \quad 24.9$ & 8 & I6 $3332.9^{8}$ & $9.73^{2}$ & $+5^{6} 5422.3$ & 0.630 & 2 \\
\hline
\end{tabular}

The planetary perturbations being small have not as yet been taken into account as $I$ intend to make a more

\begin{tabular}{|c|c|c|c|c|c|}
\hline * & \multicolumn{2}{|c|}{$\alpha$ I 883.0} & \multicolumn{2}{|c|}{$\delta 1883.0$} & A utorität \\
\hline I & $16^{\text {h }} 30^{\text {in }} \quad 6^{8} 47$ & -0.65 & $+57^{\circ} 44^{\prime} 66^{\prime \prime} 5$ & +16.5 & Helsingfors. Zonen 3 Beob. \\
\hline 2 & $16 \quad 32 \quad 23.93$ & -0.65 & $+5^{6} 4341.4$ & +16.0 & $3 \gg$ \\
\hline
\end{tabular}
complete discussion of the comet when all the observations have been published.

\section{Beobachtungen des Cometen Pons-Brooks.}

Auf der Sternwarte in Helsingfors.

Die folgenden 2 Beobachtungen habe ich mit dem Ringmicrometer des 7-zölligen Aequatoreals der Stern warte erhalten:

Mittlere Oerter der Vergleichsterne für $\mathbf{1} 883.0$.

Helsingfors 1883 Oct. r 6 .

A. Donner.

\section{Aufforderung betr. Beobachtungen des Cometen 1881 VIII (entd. von Swift Nov. 16).}

Herr S. Oppenheim, Assistent der Sternwarte in Wien, ist mit einer definitiven Bahnbestimmung des obigen Cometen beschäftigt und ersucht höflichst diejenigen Astronomen, welche im Besitze noch nicht publicirter Beobachtungen sind, ihm dieselben gef. direct baldigst zusenden zu wollen.

$K r$.

\section{Inhalt:}

Zu Nr. 2549-50. Haupt. Die Ausgleichung grosser geodätischer Dreiecke. 65. - C. H. F. Peters. Observations of the Great Comel of 1882.85 . -W. Wislicenus. Constellation der Jupiterstrabanten 1883 Oct. 14. 87. - L. Wizek. Beobachtungen auf der k. k. Ster!warte in Prag. 87.-- F. F. Parson. Elliptic Elements of Comet 1882 I. 91. - A. Donner. Beobachtungen des Cometen Pons-Brooks. 95. - Aufforderung betr. Beobachtungen des Cometen I88I VIII. 95. 